for all $t>T+\tau$. Since $T+\tau$ is independent of $n$ it follows, by (3), that $s_{n}$ is well distributed mod 1 .

\title{
REFERENCES
}

1. J. W. S. Cassels, An introduction to diophantine approximation, Cambridge University Press, 1959, Chapter IV, §5.

2. J. F. Koksma, Diophantische Approximationen, Ergebnisse der Mathematik, vol. IV, Berlin, Springer, 1937, Chapter VIII, \$3.

3. G. M. Petersen, Almost convergence and uniformly distributed sequences, Quart. J. Math. vol. 7 (1956) pp. 188-191.

4. H. Weyl, Über die Gleichverteilung von Zahlen mod Eins, Math. Ann. vol. 77 (1916) pp. 313-352.

BedFord College, London

\section{THE EXISTENCE OF NONABSOLUTELY CONVERGENT FOURIER SERIES ON COMPACT GROUPS}

GUSTAVE RABSON

1. I. E. Segal has proved [1] that on a locally compact abelian group with infinitely many elements the class of complex-valued functions which are absolutely convergent Fourier transforms is a dense set of the first category in the space of complex-valued continuous functions vanishing at infinity. Although he assumes throughout that the group is abelian, his Lemma 5, which states the result for compact metric abelian groups, applies as well without assuming the group to be abelian with only minor changes in the wording of the proof. The assumption that the group is metric can then be eliminated by using the fact that any compact infinite group has a compact infinite second countable factor group (which is easy to prove using the fact that there are unitary representations separating every pair of points) and then proceeding as we do in the final steps of the proof of our main theorem.

In this paper we shall prove that on every compact group, not of dimension zero, there is a continuous complex-valued function which is not an absolutely convergent Fourier transform. This theorem is weaker than Segal's since the fact that the group is not of dimension zero seems to be intrinsic in the proof and we conclude nothing about the category of the set of all absolutely convergent Fourier transforms. However, the proof being entirely different from Segal's, and being constructive, may be of some interest. The author is indebted

Received by the editors September 24, 1958 and, in revised form, March 5, 1959. 
to Professor H. Samelson for many helpful discussions on this subject. The manuscript was prepared in partial fulfillment of a contract with the National Science Foundation.

2. The idea behind the proof is that on every compact Lie group there is a circular subgroup on which we may construct a function whose Fourier series (on the circular subgroup) does not converge uniformly. This function may then be extended to the entire group in such a way that the Fourier series of the extended function does not converge absolutely. We then extend the result to arbitrary compact groups, not of dimension zero, by using the fact that every such group may be approximated by Lie groups.

3. Let $\Gamma$ be a compact group and $G$ a closed subgroup of $\Gamma$. Let $\left\{\chi_{\alpha}\right\}$ and $\left\{x_{a}\right\}$ be the characters of the irreducible unitary representations of $\Gamma$ and $G$ respectively. Let $\Phi$ be a central function in $L^{2}(\Gamma)$ and $F$ the restriction of $\Phi$ to $G$. It is known that the characters of the irreducible unitary representations of $\Gamma$ form a complete orthonormal system of functions in the space of central functions of $L^{2}(\Gamma)$ [4, p. 85]. By the Fourier series of a central function, $\Phi$, we mean $\sum_{\alpha}\left(\Phi, \chi_{\alpha}\right) \chi_{\alpha}$. The order in which we take the terms will be immaterial in what follows. If the group does not satisfy the second axiom of countability then $\left(\Phi, \chi_{\alpha}\right)=0$ except on a finite or countable set because of Parseval's theorem. By $\int_{\Gamma} d \xi$ and $\int_{G} d x$ we mean the Haar integrals on $\Gamma$ and $G$ respectively, normalized so that $\int_{\Gamma} d \xi=\int_{G} d x=1$. Let $^{1}(\alpha: a)=\int_{G} \chi_{\alpha}(x)\left(X_{a}(x)\right)^{c} d x$. Since every representation of $\Gamma$, when restricted to $G$, is also a representation of $G$ it follows that $\chi_{\alpha}$, when restricted to $G$, will be the character of a representation of $G$. Consequently it may be written as the sum of a finite number of the characters of $G$. It follows from the orthogonality of the characters that $(\alpha: a)$ is the number of times that $X_{a}$ appears as a summand of $\chi_{\alpha}$ restricted to $G$.

Lemma 1. If $\Phi$ is a central function of $L^{2}(\Gamma)$ whose Fourier series converges absolutely at the identity, $\epsilon$, then the Fourier series of $\Phi$ converges absolutely at every point and converges to $\Phi$ uniformly.

From the fact that the representations are unitary it follows that for every $\xi \in \Gamma,\left|\chi_{\alpha}(\xi)\right| \leqq \chi_{\alpha}(\epsilon)$. Consequently,

$$
\left|\left(\Phi, \chi_{\alpha}\right) \chi_{\alpha}(\xi)\right| \leqq\left|\left(\Phi, \chi_{\alpha}\right) \chi_{\alpha}(\epsilon)\right| \text {. }
$$

It follows that $\sum_{\alpha}\left(\Phi, \chi_{\alpha}\right) \chi_{\alpha}(\xi)$ converges absolutely and uniformly. The fact that it converges to $\Phi$ follows as for classical Fourier series.

1 ( ) denotes the complex conjugate of the expression in parentheses. 
Lemma 2. Let $\Phi$ satisfy the hypothesis of Lemma 1 and $F$ be $\Phi$ restricted to $G$, then $\int_{G} F(x)\left(X_{a}(x)\right)^{c} d x=\sum_{\alpha}(\alpha: a) \int_{\Gamma} \Phi(\xi)\left(\chi_{\alpha}(\xi)\right)^{c} d \xi$.

This lemma appears in $[4$, p. 87]. We include the proof here for completeness.

By Lemma 1 we have:

$$
F(s)=\sum_{\alpha} \chi_{\alpha}(s) \int_{\Gamma} \Phi(\xi)\left(\chi_{\alpha}(\xi)\right)^{c} d \xi
$$

for all $s \in G$, the convergence being uniform. So we have for the Fourier coefficients of $F$ :

$$
\begin{aligned}
\int_{G} F(s)\left(X_{a}(s)\right)^{c} d s & =\sum_{\alpha} \int_{G} \chi_{\alpha}(s)\left(X_{a}(s)\right)^{c} d s \cdot \int_{\Gamma} \Phi(\xi)\left(\chi_{\alpha}(\xi)\right)^{c} d \xi \\
& =\sum_{\alpha}(\alpha: a) \int_{\Gamma} \Phi(\xi)\left(\chi_{\alpha}(\xi)\right)^{c} d \xi .
\end{aligned}
$$

The interchange of the order of the summation and integration is justified by the uniform convergence of (1).

Lemma 3. The Fourier series of $F$ (assumed to satisfy the same conditions as in Lemma 2) converges to it absolutely at the identity, e, of $G$.

Proof.

$$
\begin{aligned}
\sum_{a} X_{a}(e) \int_{G} F(x)\left(X_{a}(x)\right)^{c} d x & =\sum_{a} \sum_{\alpha}(\alpha: a) X_{a}(e) \int_{\Gamma} \Phi(\xi)\left(\chi_{\alpha}(\xi)\right)^{c} d \xi \\
& =\sum_{\alpha} \sum_{a}(\alpha: a) X_{a}(e) \int_{\Gamma} \Phi(\xi)\left(\chi_{\alpha}(\xi)\right)^{c} d \xi \\
& =\sum_{\alpha} \chi_{\alpha}(e) \int_{\Gamma} \Phi(\xi)\left(\chi_{\alpha}(\xi)\right)^{c} d \xi .
\end{aligned}
$$

The last sum converges absolutely by hypothesis. The interchange of the order of summation is justified by the fact that

$$
\sum_{\alpha} \sum_{a}\left|(\alpha: a) X_{a}(e) \int_{\Gamma}\left(\chi_{\alpha}(\xi)\right)^{c} \Phi(\xi) d \xi\right|=\sum_{\alpha}\left|\int_{\Gamma}\left(\chi_{\alpha}(\xi)\right)^{c} \Phi(\xi) d \xi \cdot \chi_{\alpha}(\epsilon)\right|
$$

and this last series is assumed to be convergent so both summations are simply rearrangements of an absolutely convergent series.

LEMma 4. On any compact Lie group, not of dimension zero, there is a continuous central function whose Fourier series is not absolutely convergent. 
The proof makes use of some of the ideas concerning maximal abelian subgroups of a Lie group [2] and [3].

Proof. Let $T$ be a maximal abelian toroidal subgroup of the Lie group $G$. The group of automorphisms of $T$ of the form $s T s^{-1}$, for $s \in G$, form a finite group, $\psi$, of isomorphisms of $T$ with itself. The group $\psi$ is generated by reflections in a set of hyperplanes (the diagram of the group). These hyperplanes consist of the singular elements. An element of $T$ is called singular if it lies on some toroidal group other than $T$. If there is an $s$ such that $s t s^{-1}=t^{\prime}$ for $t$ and $t^{\prime}$ in $T$ then, since $s T s^{-1}$ is a toroidal group, either $s T s^{-1}=T$ or $t$ and $t^{\prime}$ are singular. In a fundamental domain of this group we can find a circular subgroup of $G$. On this circular subgroup we define a function, $\phi$, whose Fourier series does not converge absolutely at the identity, $e$. This function can then be extended to a continuous function on the fundamental domain which vanishes on the hyperplane of singular elements. This extension can then be further extended by means of the group $\psi$ to a continuous function on $T$ which is invariant with respect to $\psi$. We finally define the function $\phi^{*}$ on $G$, by means of the equation $\phi^{*}(x)=\phi(t)$ where $x=s t s^{-1}, x, s \in G$ and $t \in T$ (we use the fact that every element of $G$ is an automorph of some element of $T$ ). If $x=s t s^{-1}=s^{\prime} t^{\prime}\left(s^{\prime}\right)^{-1}$ then $t=\left(s^{-1} s^{\prime}\right) t^{\prime}\left(s^{-1} s^{\prime}\right)^{-1}$ and $\phi(t)=\phi\left(t^{\prime}\right)$.

Now $\phi^{*}$ is continuous. To prove this consider any closed set, $K$, of complex numbers. $\left(\phi^{*}\right)^{-1}(K)$ is a closed set on the torus. But the set of elements of $G$ which have conjugates in $\left(\phi^{*}\right)^{-1}(K)$ is also a closed set, for if $\left\{\gamma_{\alpha}\right\}$ is a set of elements of $G$, each of which has a conjugate, $x_{\alpha} \gamma_{\alpha} x_{\alpha}^{-1}$ in $\left(\phi^{*}\right)^{-1}(K)$ then the $x_{\alpha}$ have a limit point $x$ and, if $\gamma$ is a limit point of the $\gamma_{\alpha}, x \gamma x^{-1}$ will be a limit point of $\left\{x_{\alpha} \gamma_{\alpha} x_{\alpha}^{-1}\right\}$ and so will also be in $\left(\phi^{*}\right)^{-1}(K) . \phi^{*}$ is a central function since for any $\gamma$ and $s$ in $G$ there is an $x$ in $G$ and a $t$ in $T$ such that $\gamma=x t x^{-1}$ and so we have

$$
\phi^{*}\left(s \gamma s^{-1}\right)=\phi^{*}\left(s x t x^{-1} s^{-1}\right)=\phi^{*}(t)=\phi^{*}\left(x t x^{-1}\right)=\phi^{*}(\gamma) .
$$

Consequently, by Lemma 3, the Fourier series of $\phi^{*}$ cannot converge absolutely.

THEOREM. On any compact group of positive dimension there is a continuous central function whose Fourier series does not converge absolutely.

We may assume that $G$ is connected, otherwise we can deal with the component of the identity and extend the function to a continuous central function on $G$ by setting it equal to 0 on the components other than that containing the identity. 
Let $K$ be the kernel of a nontrivial unitary representation of $G$. $G / K$ is nontrivial, connected, and, being isomorphic to a closed subgroup of a unitary group, it must be a Lie group of dimension at least 1. Every irreducible representation, $M$, of $G / K$ induces an irreducible representation, $M^{\prime}$, of $G$, in the following manner. If $\pi$ is the natural map from $G$ onto $G / K$ we define $M^{\prime}(g)$ to be equal to $M(\pi(g))$ for every $g$ in $G$. Consequently every irreducible character, $\chi$, of $G / K$, induces an irreducible character $\chi^{\prime}$ of $G$. Consider a central function $F$ on $G / K$ whose Fourier series does not converge absolutely. This induces the function $F^{\prime}$ on $G$ defined by $F^{\prime}(g)=F(\pi(g))$ for each $g$ in $G$. The functions $F$ and $F^{\prime}$ are both central functions on their respective groups and so may be expanded with respect to the characters. The coefficients of the character series of $F^{\prime}$ will be $\int_{G} F^{\prime}(x)\left(\chi_{\alpha}^{\prime}(x)\right)^{c} d x$. Since for any function $\phi$ on $G / K$ we may define an invariant integral by the formula $\int_{G / K} \phi(x) d x=\int_{G} \phi^{\prime}(x) d x$ and since the Haar measure is unique it follows that for any character $\chi_{a}$ on $G / K$,

$$
\int_{G / K} F(x)\left(\chi_{a}(x)\right)^{c} d x=\int_{G} F^{\prime}(x)\left(\chi_{a}^{\prime}(x)\right)^{c} d x .
$$

Consequently the character series of $F$ consists of a subset of the terms of the character series of $F^{\prime}$. If the latter converged absolutely, so would the former. However, $F$ was constructed so that its character series does not converge absolutely.

\section{REFERENCES}

1. I. E. Segal, The class of functions which are absolutely convergent Fourier transforms, Acta Sci. Math. Szeged, Pars B vol. 12 (1950) pp. 157-161.

2. E. Stiefel, Über eine Beziehung zwischen geschlossenen Lie'schen Gruppen und diskontinuierlichen Bewegungsgruppen euklidischer Räume und ihre Anwendung auf die Aufzählung der einfachen Lie'schen Gruppen. Comment, Math. Helv. vol. 14 (19411942) pp. 350-380.

3. - Kristallographische Bestimmung der Charaktere der geschlossenen Lie'schen Gruppen, Comment. Math. Helv. vol. 17 (1944-1945) pp. 165-200. 1940.

4. A. Weil, L'intégration dans les groupes topologiques et ses applications, Paris,

Antioch COLlege AND

UNIVERSITY OF MiCHIGAN 